\title{
EVENTUAL DISCONJUGACY OF A LINEAR DIFFERENTIAL EQUATION
}

\author{
WILLIAM F. TRENCH
}

\begin{abstract}
A sufficient condition is given for eventual disconjugacy of an $n$th order linear differential equation.
\end{abstract}

1. Introduction. If there is an interval $[a, \infty)$ on which no nontrivial solution of the scalar equation

$$
y^{(n)}+p_{1}(t) y^{(n-1)}+\cdots+p_{n}(t) y=0
$$

has more than $n-1$ zeros, counting multiplicities, then (1) is said to be eventually disconjugate. From a special case of a theorem of Willett [4], (1) is eventually disconjugate if $p_{1}, \ldots, p_{n} \in C[0, \infty)$ and

$$
\int^{\infty} t^{k-1}\left|p_{k}(t)\right| d t<\infty, \quad 1 \leqslant k \leqslant n .
$$

By an easy argument based on Polya's disconjugacy condition [2], the author showed in [3] that (1) is eventually disconjugate if it has a fundamental system $\left\{y_{0}, \ldots, y_{n-1}\right\}$ such that, for $0 \leqslant m \leqslant n-1$,

$$
y_{m}^{(r)}(t)= \begin{cases}t^{m-r}(1+o(1)) /(m-r) !, & 0 \leqslant r \leqslant m, \\ o\left(t^{m-r}\right), & m+1 \leqslant r \leqslant n-1 .\end{cases}
$$

By a theorem of Hartman and Wintner [1], (2) implies that (1) has such a fundamental system. In [3] the author gave a condition weaker than (2) which implies the same conclusion, but requires the existence of certain auxiliary functions which may not be easy to find. Here we give a condition which is weaker than (2) and imposes only readily verifiable requirements on $p_{1}, \ldots, p_{n}$.

2. A preliminary lemma. To state and prove our main results, we need the following lemma.

Lemma 1. Suppose $p \in C(0, \infty)$ and $\int^{\infty} t^{k-1} p(t) d t$ converges (perhaps conditionally). Define

$$
I_{0}(t ; p)=p(t)
$$

and

$$
I_{j}(t ; p)=\int_{t}^{\infty} I_{j-1}(s ; p) d s=\int_{t}^{\infty} \frac{(s-t)^{j-1}}{(j-1) !} p(s) d s, \quad 1 \leqslant j \leqslant k .
$$

Received by the editors September 8, 1982 and, in revised form, January 3, 1983.

1980 Mathematics Subject Classification. Primary 34C10.

(C)1983 American Mathematical Society $0002-9939 / 83 \$ 1.00+\$ .25$ per page 
Then the integrals (5) converge and satisfy the inequalities

$$
\left|I_{j}(t ; p)\right| \leqslant \frac{2 \delta(t) t^{j-k}}{(j-1) !}, \quad 1 \leqslant j \leqslant k
$$

where

$$
\delta(t)=\sup _{T \geqslant t}\left|\int_{T}^{\infty} s^{k-1} p(s) d s\right|=o(1)
$$

The integrals

$$
\int^{\infty} t^{k-j-1} I_{j}(t ; p) d t, \quad 0 \leqslant j \leqslant k-1,
$$

all converge, and if this convergence is absolute for some $j_{0}$ in $\{0,1, \ldots, k-1\}$, then it is absolute for $j_{0} \leqslant j \leqslant k-1$.

Proof. Our assumption and Abel's integral test imply that $\int^{\infty} s^{r} p(s) d s$ converges for $0 \leqslant r \leqslant k-1$; therefore, $I_{j}(t ; p)$ converges for $1 \leqslant j \leqslant k$. With $U(t)=$ $\int_{t}^{\infty} s^{k-1} p(s) d s$,

$$
\int_{t}^{\infty}(s-t)^{j-1} p(s) d s=-\int_{t}^{\infty}\left(1-\frac{t}{s}\right)^{j-1} s^{j-k} U^{\prime}(s) d s
$$

If $2 \leqslant j \leqslant k$, integrating the right side by parts yields

$$
\int_{t}^{\infty}(s-t)^{j-1} p(s) d s=\int_{t}^{\infty} U(s) \frac{d}{d s}\left[\left(1-\frac{t}{s}\right)^{j-1} s^{j-k}\right] d s .
$$

This and routine estimates yield (6) for $2 \leqslant j \leqslant k$, since $|U(s)| \leqslant \delta(t)$ and

$$
\left|\frac{d}{d s}\left[\left(1-\frac{t}{s}\right)^{j-1} s^{j-k}\right]\right| \leqslant(k-j) s^{j-k-1}+t^{j-k} \frac{d}{d s}\left(1-\frac{t}{s}\right)^{j-1}
$$

if $s \geqslant t$ and $k \geqslant j$. (In fact, we may drop the 2 in (6) if $j=k$.) If $j=1$, integrating the right side of (9) by parts yields

$$
\int_{t}^{\infty} p(s) d s=t^{-k+1} U(t)-(k-1) \int_{t}^{\infty} s^{-k} U(s) d s,
$$

which implies (6) with $j=1$.

From (4) and (5), integration by parts yields

$$
\int_{t_{1}}^{t_{2}} t^{k-j-1} I_{j}(t ; p) d t=\left.\frac{t^{k-j}}{k-j} I_{j}(t ; p)\right|_{t_{1}} ^{t_{2}}+\frac{1}{k-j} \int_{t_{1}}^{t_{2}} t^{k-j} I_{j-1}(t ; p) d t
$$

if $1 \leqslant j \leqslant k-1$; hence, (6) and the convergence of (8) for $j=0$ imply that the integrals in (8) all converge, by finite induction. If

$$
\int^{\infty} t^{k-j-1}\left|I_{j}(t ; p)\right| d t<\infty
$$

for some $j<k-1$, then

$$
\int_{t}^{\infty}\left|I_{j}(s ; p)\right| d s=o\left(t^{-k+j+1}\right) .
$$


Now,

$$
\begin{aligned}
\int_{t_{1}}^{t_{2}} t^{k-j-2}\left(\int_{t}^{\infty}\left|I_{j}(s ; p)\right| d s\right) d t= & \left.\frac{t^{k-j-1}}{k-j-1} \int_{t}^{\infty}\left|I_{j}(s ; p)\right| d s\right|_{t_{1}} ^{t_{2}} \\
& +\frac{1}{k-j-1} \int_{t_{1}}^{t_{2}} t^{k-j-1}\left|I_{j}(t ; p)\right| d t .
\end{aligned}
$$

Therefore, (10) and (11) imply that

$$
\int^{\infty} t^{k-j-2}\left(\int_{t}^{\infty}\left|I_{j}(s ; p)\right| d s\right) d t<\infty
$$

which in turn implies that

$$
\int^{\infty} t^{k-j-2}\left|I_{j+1}(t ; p)\right| d t<\infty
$$

since

$$
\left|I_{j+1}(t ; p)\right| \leqslant \int_{t}^{\infty}\left|I_{j}(s ; p)\right| d s
$$

(See (5) with $j$ replaced by $j+1$.) This completes the proof.

To see that Lemma 1 has nontrivial applications, let

$$
Q(t)=t^{-k} \exp \left[i|\log t|^{\gamma+1}\right] \quad(\gamma>0) .
$$

The substitution $x=(\log t)^{\gamma+1}$ shows that if $F$ is a polynomial with purely real or purely imaginary nonzero constant term, then the real and imaginary parts of

$$
\int^{\infty} t^{k-1}(\log t)^{-j \gamma} F\left((\log t)^{-\gamma}\right) Q(t) d t
$$

converge conditionally if $0 \leqslant j \gamma \leqslant 1$, or absolutely if $j \gamma>1$. Moreover, if $t>1$ and $0 \leqslant j \leqslant k-1$, then

$$
I_{j}(t ; Q)=t^{j}(\log t)^{-j \gamma} F_{j}\left((\log t)^{-\gamma}\right) Q(t)+O\left(t^{-k+j}(\log t)^{-k \gamma}\right) .
$$

where $F_{j}$ is a polynomial of the kind mentioned above. (From (4), (13) holds with $j=0$. If $0 \leqslant r \leqslant k-2$ and (13) holds with $j=r$, then it can be established for $j=r+1$ by means of the first equality in (5) and repeated integration by parts, integrating the exponential factor at each step. Therefore, (13) holds for $0 \leqslant j \leqslant k-$ 1 , by finite induction.) If $j_{0} \geqslant 1$ and $1 / j_{0}<\gamma<1 /\left(j_{0}-1\right)$, then multiplying the " 0 " term in (13) by $t^{k-j-1}$ produces an absolutely integrable term (since $k \gamma>1$ ). Therefore, (13) and the stated convergence properties of (12) imply that the real and imaginary parts of $\int^{\infty} t^{k-j-1} I_{j}(t ; Q) d t$ converge conditionally if $0 \leqslant j \leqslant j_{0}-1$, or absolutely if $j_{0} \leqslant j \leqslant k-1$. Hence, if

$$
p(t)=t^{-k} \cos \left[\mid \log t \gamma^{\gamma+1}\right] \text { or } p(t)=t^{-k} \sin \left[|\log t|^{\gamma+1}\right],
$$

then $\int^{\infty} t^{k-j-1} I_{j}(t ; p) d t$ has these properties. 
3. Main results. The following is our main theorem.

TheOREM 1. Suppose $p_{1}, \ldots, p_{n} \in C(0, \infty)$. Let the integrals

$$
\int^{\infty} t^{k-1} p_{k}(t) d t, \quad 1 \leqslant k \leqslant n,
$$

converge, and

$$
\int^{\infty}\left|I_{k-1}\left(t ; p_{k}\right)\right| d t<\infty, \quad 1 \leqslant k \leqslant n .
$$

Then (1) is eventually disconjugate.

Theorem 1 follows from the next theorem, by the argument given in [3].

THEOREM 2. The assumptions of Theorem $1 \mathrm{imply}$ that (1) has a fundamental system $\left\{y_{0}, \ldots, y_{n-1}\right\}$ which satisfies (3).

Proof. Let $m$ be a fixed integer, $0 \leqslant m \leqslant n-1$. (Some quantities below depend upon $m$, but we will not make this explicit in the notation.) For $t_{0}>0$, let $B\left(t_{0}\right)$ be the Banach space of functions $y$ in $C^{(n-1)}\left[t_{0}, \infty\right)$ such that

$$
y^{(r)}(t)=O\left(t^{m-r}\right), \quad 0 \leqslant r \leqslant n-1,
$$

with norm

$$
\|y\|=\sup _{t \geqslant t_{0}}\left\{\sum_{r=0}^{n-1} t^{r-m}\left|y^{(r)}(t)\right|\right\} .
$$

Let $M y=\sum_{k=1}^{n} p_{k} y^{(n-k)}$. We first show that the transformation $T$ defined by

$$
(T y)(t)=1+\int_{t}^{\infty} \frac{(t-s)^{n-1}}{(n-1) !}(M y)(s) d s \quad \text { if } m=0
$$

or by

$$
(T y)(t)=\frac{t^{m}}{m !}+\int_{t_{0}}^{t} \frac{(t-\lambda)^{m-1}}{(m-1) !} d \lambda \int_{\lambda}^{\infty} \frac{(\lambda-s)^{n-m-1}}{(n-m-1) !}(M y)(s) d s
$$

if $m=1, \ldots, n-1$, is a contraction mapping of $B\left(t_{0}\right)$ into itself if $t_{0}$ is sufficiently large. Consider the integral

$$
J(t ; h)=\int_{t}^{\infty} s^{n-m-1}(M h)(s) d s, \quad h \in B\left(t_{0}\right), t \geqslant t_{0} .
$$

Using (5) and repeated integration by parts yields

$$
\begin{aligned}
\int_{t}^{\bar{t}} s^{n-m-1}(M h)(s) d s= & \sum_{k=1}^{n} \int_{t}^{\bar{t}} s^{n-m-1} p_{k}(s) h^{(n-k)}(s) d s \\
= & -\left.\sum_{k=2}^{n} \sum_{j=1}^{k-1} I_{j}\left(s ; p_{k}\right)\left[s^{n-m-1} h^{(n-k)}(s)\right]^{(j-1)}\right|_{t} ^{t} \\
& +\int_{t}^{\bar{t}}\left(\sum_{k=1}^{n} I_{k-1}\left(s ; p_{k}\right)\left[s^{n-m-1} h^{(n-k)}\right]^{(k-1)}\right) d s .
\end{aligned}
$$


By using Liebniz' formula for the derivatives of a product, and rearranging terms, we can rewrite the integrand in the last member of (19) as

$$
\sum_{j=0}^{n-m-1}\left[s^{n-m-1}\right]^{(j)} h^{(n-j-1)}(s) \sum_{k=j+1}^{n}\left(\begin{array}{c}
k-1 \\
j
\end{array}\right) I_{k-1}\left(s ; p_{k}\right) .
$$

Therefore, (14) implies that this integral converges absolutely at $\bar{t} \rightarrow \infty$, because

$$
\left|\left[s^{n-m-1}\right]^{(j)} h^{(n-j-1)}(s)\right| \leqslant K_{j}\|h\|, \quad 0 \leqslant j \leqslant n-m-1,
$$

where $K_{j}$ is a universal constant (see (1.5)). Moreover, from (6) with $p=p_{k}$,

$$
\left|I_{j}\left(t ; p_{k}\right)\right| \leqslant \frac{2 \delta_{k}(t) t^{j-k}}{(j-1) !}, \quad 1 \leqslant j \leqslant k,
$$

with $\delta_{k}$ as in (7) with $p=p_{k}$. From (15),

$$
\left|\left[s^{n-m-1} h^{(n-k)}(s)\right]^{(j-1)}\right| \leqslant B_{j k}\|h\| t^{k-j}, \quad 1 \leqslant j \leqslant k-1,
$$

where $B_{j k}$ is a universal constant. Therefore, letting $t \rightarrow \infty$ in (19) and applying (14), (20), (21) and (22) shows that (18) converges and

$$
|J(t ; h)| \leqslant \sigma(t)\|h\|, \quad t \geqslant t_{0},
$$

where

$$
\begin{aligned}
\sigma(t)= & 2 \sum_{k=2}^{n}\left(\sum_{j=1}^{k-1} \frac{B_{j k}}{(j-1) !}\right) \delta_{k}(t) \\
& +\sum_{j=0}^{n-m-1} K_{j} \int_{t}^{\infty}\left|\sum_{k=j+1}^{n}\left(\begin{array}{c}
k-1 \\
j
\end{array}\right) I_{k-1}\left(s ; p_{k}\right)\right| d s .
\end{aligned}
$$

Since $J(t ; h)$ converges, Lemma 1 with $p=M h$ and $k=n-m$ implies that the function $\hat{h}$ defined by

$$
\hat{h}(t)=\int_{t}^{\infty} \frac{(t-s)^{n-1}}{(n-1) !}(M h)(s) d s \quad \text { if } m=0,
$$

or by

$$
\hat{h}(t)=\int_{t_{0}}^{t} \frac{(t-\lambda)^{m-1}}{(m-1) !} d \lambda \int_{\lambda}^{\infty} \frac{(\lambda-s)^{n-m-1}}{(n-m-1) !}(M h)(s) d s
$$

if $m=1, \ldots, n-1$, is defined for $t \geqslant t_{0}$. We will now show that $\hat{h} \in B\left(t_{0}\right)$, and estimate $\|\hat{h}\|$. Differentiating (24) or (25) yields

$$
\hat{h}^{(r)}(t)=\int_{t}^{\infty} \frac{(t-s)^{n-r-1}}{(n-r-1) !}(M h)(s) d s, \quad m \leqslant r \leqslant n-1,
$$

so Lemma 1 and (23) imply that

$$
\left|\hat{h}^{(r)}(t)\right| \leqslant \frac{2\|h\| \sigma(t) t^{m-r}}{(n-r-1) !}, \quad m \leqslant r \leqslant n-1 .
$$


If $m \geqslant 1$, then we must also estimate $\hat{h}^{(r)}(t), 0 \leqslant r \leqslant m-1$. From (25) and (26) (the latter with $r=m$ ),

$$
\hat{h}(t)=\int_{t_{0}}^{t} \frac{(t-\lambda)^{m-1}}{(m-1) !} \hat{h}^{(m)}(\lambda) d \lambda .
$$

Differentiating this and invoking (27) with $r=m$ yields

$$
\left|\hat{h}^{(r)}(t)\right| \leqslant \frac{2\|h\|}{(m-r-1) !(n-m-1) !} \int_{t_{0}}^{t}(t-\lambda)^{m-r-1} \sigma(\lambda) d \lambda,
$$

$0 \leqslant r \leqslant m-1$. Since $\sigma$ is nonincreasing, this implies that

$$
\left|\hat{h}^{(r)}(t)\right| \leqslant \frac{2\|h\| \sigma\left(t_{0}\right) t^{m-r}}{(m-r) !(n-m-1) !}, \quad 0 \leqslant r \leqslant m-1 .
$$

Now (27) and (29) imply that $\hat{h} \in B\left(t_{0}\right)$. From (16) and (24) or (17) and (25), this implies that $T y \in B\left(t_{0}\right)$ if $y \in B\left(t_{0}\right)$. Moreover, if $y$ and $\tilde{y}$ are in $B\left(t_{0}\right)$, then (27) and (29) with $h=y-\tilde{y}$ imply that $\|T y-T \tilde{y}\| \leqslant K \sigma\left(t_{0}\right)\|y-\tilde{y}\|$, where $K$ is a universal constant. Now choose $t_{0}$ so that $K \sigma\left(t_{0}\right)<1$. Then $T$ is a contraction mapping of $B\left(t_{0}\right)$ into itself, and therefore $T$ has a fixed point (function) $y_{m}$ such that, for $t \geqslant t_{0}$.

$$
y_{0}(t)=1+\int_{t}^{\infty} \frac{(t-s)^{n-1}}{(n-1) !}\left(M y_{0}\right)(s) d s \quad \text { if } m=0
$$

(see (16)), or

$$
y_{m}(t)=\frac{t^{m}}{m !}+\int_{t_{0}}^{t} \frac{(t-\lambda)^{m-1}}{(m-1) !} d \lambda \int_{\lambda}^{\infty} \frac{(\lambda-s)^{n-m-1}}{(n-m-1) !}\left(M y_{m}\right)(s) d s
$$

if $m=1, \ldots, n-1$ (see (17)). Clearly, $y_{0}, \ldots, y_{n-1}$ all satisfy $(1)$ on $\left(t_{0}, \infty\right)$, and they can be extended over $(0, \infty)$ as solutions of (1). If $h=y_{m}$ and $\hat{h}$ is the integral on the right of (30) or (31) (see (24) and (25)), then (27) implies (3) for $m \leqslant r \leqslant n-1$. If $m \geqslant 1$, then (28) implies that

$$
t^{r-m}\left|h^{(r)}(t)\right| \leqslant \frac{2\|h\| t^{-1}}{(m-r-1) !(n-m-1) !} \int_{t_{0}}^{t} \sigma(\lambda) d \lambda, \quad 0 \leqslant r \leqslant m-1,
$$

which implies (3) for $0 \leqslant r \leqslant m-1$, since the right side here approaches zero as $t \rightarrow \infty$. (This is obvious if $\int^{\infty} \sigma(\lambda) d \lambda<\infty$; if $\int^{\infty} \sigma(\lambda) d \lambda=\infty$, then it follows from l'Hospital's rule, since $\lim _{t \rightarrow \infty} \sigma(t)=0$.) This completes the proof of Theorem 2 .

\section{REFERENCES}

1. P. Hartman and A. Wintner, Asymptotic integration of linear differential equations, Amer. J. Math. 77 (1955), 45-87.

2. G. Polya, On the mean-value theorem corresponding to a given linear homogeneous differential equation, Trans. Amer. Math. Soc. 24 (1924), 312-324.

3. W. F. Trench, A sufficient condition for eventual disconjugacy, Proc. Amer. Math. Soc. 52 (1975), 139-146.

4. D. Willett, Disconjugacy tests for singular linear differential equations, SIAM J. Math. Anal. 2 (1971), 536-545.

Department of Mathematical Sciences, Drexel University, Philadelphia, Pennsylvania 19104 